\title{
Search for electroweak single top quark production with CDF
}

\section{Yves Kemp ${ }^{* \dagger}$}

Universität Karlsruhe, Germany

E-mail: kemp@ekp.physik.uni-karlsruhe.de

We report on a search for Standard Model t-channel and s-channel single top quark production in $p \bar{p}$ collisions at a center of mass energy of $1.96 \mathrm{TeV}$. We use a data sample corresponding to $162 \mathrm{pb}^{-1}$ recorded by the upgraded Collider Detector at Fermilab. We find no significant evidence for electroweak top quark production and set upper limits at the $95 \%$ confidence level on the production cross section, consistent with the Standard Model: $10.1 \mathrm{pb}$ for the t-channel, $13.6 \mathrm{pb}$ for the s-channel and $17.8 \mathrm{pb}$ for the combined cross section of $\mathrm{t}$ - and s-channel.

HEP2005 International Europhysics Conference on High Energy Physics

21-27 Jul 2005

Lisboa, Portugal

* Speaker.

† On behalf of the CDF Collaboration 
At the Tevatron, the most important production mode for top quarks is the strong interaction, where top quarks are produced in pairs. Top quarks can also be produced via electroweak interactions, where they are then produced singly [1] ("single-top"). The two relevant single-top processes are the $\mathrm{t}$ - and the s-channel exchange of a virtual $W$ boson. The cross sections predicted by the Standard Model are $(1.98 \pm 0.25) \mathrm{pb}$ and $(0.88 \pm 0.11) \mathrm{pb}$ at $\sqrt{s}=1.96 \mathrm{TeV}$ respectively [2]. A measurement of these cross sections is of interest for the determination of $\left|V_{t b}\right|^{2}$, which could test the unitarity assumption of the CKM matrix. Single top quark production is also an important background for the search for a light Higgs boson at the Tevatron, a good understanding of single top is therefore mandatory [3].

The top quark mainly decays into a $W$ boson and a $b$ quark. For this analysis, we consider only decays of the $W$ boson into an electron or muon and the respective neutrino. The experimental signature is therefore an isolated electron or muon with $E_{T}$ respectively $p_{T}$ larger than $20 \mathrm{GeV}$, missing transverse energy larger than $20 \mathrm{GeV}$ and a jet tagged as a $b$ jet. In the s-channel, another $b$ quark can be measured as $b$ tagged jet, coming from the decay of the virtual $W$ boson. In the t-channel, the $b$ quark coming from the splitting of the gluon is rather forward, only $25 \%$ of these jets are in the fiducial volume of the detector and have a transverse energy large enough to be detected. However, the t-channel has as an additional experimental signature a light quark jet. The different objects must be fiducial: Electrons and muons are required to be $|\eta|<1.0$, the jets should be $|\eta|<2.8$. The effective $b$ tagging region is $|\eta|<1.4$ [4].

The most important backgrounds are $t \bar{t}$ quark productions, dilepton events, $W+$ jets production and QCD multijet production faking an electron or muon and missing transverse energy. The preselection cuts previously detailed remove some of these events. To increase the discrimination between signal and background, a dilepton veto and a cut on $M_{l v b}$, the mass of the top quark candidate are performed.

Table 1 summarizes the event yield obtained with a data sample corresponding to an integrated luminosity of $(162 \pm 10) \mathrm{pb}^{-1}$ taken with the upgraded Collider Detector at Fermilab (CDF II [5]). Only events in the $W+2$-jets-bin are considered. A combined search is performed where both sand t-channel are considered as signal. Furthermore, we also search separately for s- and t-channel production. To distinguish between these two channels, we consider events where exactly one jet is tagged as $b$ quark for the t-channel search and events with two jets tagged as $b$ quark for the s-channel search. An additional requirement was put on $E_{T}>30 \mathrm{GeV}$ of the leading jet for the t-channel search. We observe 42 events for the combined search, 33 and 6 for the separate search in the one and two tag bin. Considering the uncertainties, these numbers are in good agreement with predictions.

To extract the signal content in the data, we use a maximum likelihood technique. For the separate searches, we use the $Q \cdot \eta$ variable, where $Q$ is the charge of the electron respectively muon and $\eta$ the pseudorapidity of the untagged jet. We also consider the number of events in the two-tag sample. As seen in figure 1, the t-channel discriminates well against the s-channel and the background processes. The likelihood method employed includes the systematic uncertainties in the computation of the upper limits. Also, a consistent Bayesian treatment is guaranteed. Furthermore, the correlations between the different systematic uncertainties are included in the likelihood. In the combined search, we use a similar likelihood function to discriminate the two signal chan- 


\begin{tabular}{|l|c|c|c|}
\hline Process & Combined & 1-tag & 2-tag \\
\hline$t \bar{t}$ & $3.8 \pm 0.9$ & $3.2 \pm 0.7$ & $0.60 \pm 0.14$ \\
Non-Top & $30.0 \pm 5.8$ & $23.3 \pm 4.6$ & $2.59 \pm 0.71$ \\
Sum background & $33.8 \pm 5.9$ & $26.5 \pm 4.7$ & $3.19 \pm 0.72$ \\
\hline t-channel & $2.8 \pm 0.5$ & $2.7 \pm 0.4$ & $0.02 \pm 0.01$ \\
s-channel & $1.5 \pm 0.2$ & $1.1 \pm 0.2$ & $0.32 \pm 0.05$ \\
Sum single top & $4.3 \pm 0.5$ & $3.8 \pm 0.4$ & $0.34 \pm 0.05$ \\
\hline Sum expected & $38.1 \pm 5.9$ & $30.3 \pm 4.7$ & $3.53 \pm 0.72$ \\
\hline Observed & 42 & 33 & 6 \\
\hline
\end{tabular}

Table 1: Event yields with $162 \mathrm{pb}^{-1}$. Separate searches for the different single top production channels are best described by different $b$ tag bins. For the one $b$ tag bin, we demand the presence of at least on e jet with $E_{T}>30 \mathrm{GeV}$. This requirement accounts for the difference between the second column and the sum of the third and fourth column.
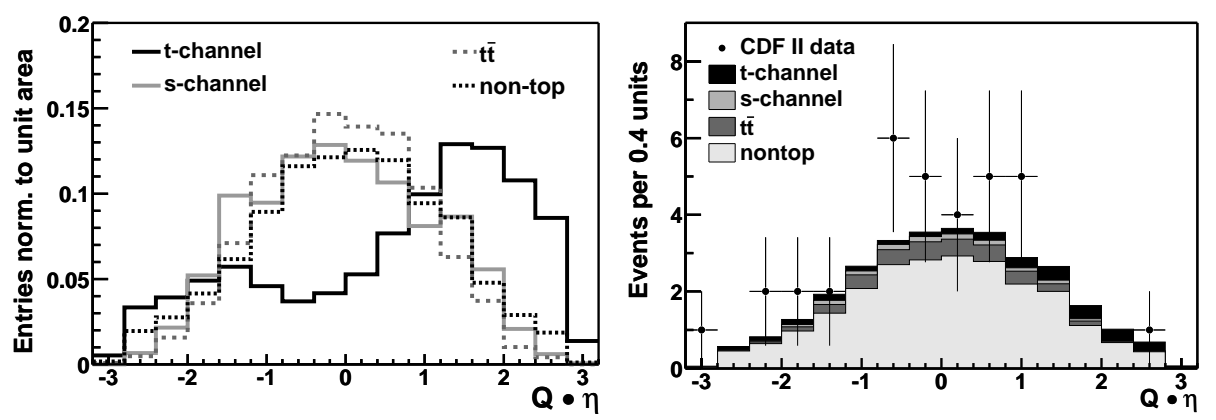

Figure 1: $Q \cdot \eta$ distributions for MC samples normalized to unit area (the distributions are the templates)(left). $Q \cdot \eta$ distributions normalized to Standard Model expectations (histograms), compared to the CDF data (points) (right).

nels against background events. The discriminating variable is $H_{T}$, the scalar sum of the missing transverse energy, the transverse energy of the electron or muon and all jets in the event. Figure 2 compares the observed data to the Standard Model prediction.

To estimate the a priori sensitivity assuming the Standard Model signal cross sections, we perform Monte Carlo pseudo experiments. The upper limit is then obtained by integrating the likelihood function. Table 2 summarizes the expected and observed limits and gives the most probable value (MPV) and highest posterior density intervals (HPD) for the single top cross sections in units of $\mathrm{pb}$. The method and the results are detailed in [6].

In summary, we find no significant evidence for electroweak top quark production in (162 \pm 10) $\mathrm{pb}^{-1}$ of integrated luminosity recorded with the CDF II detector. We set first limits for the cross sections at the Tevatron Run 2 energies.

Our plans are to further develop and expand our analysis. The event yields will be increased by including electrons in the forward region of the CDF II detector (up to $|\eta|<2$.0). We will combine more discriminating variables into a Neural Net to better discriminate between signal and 


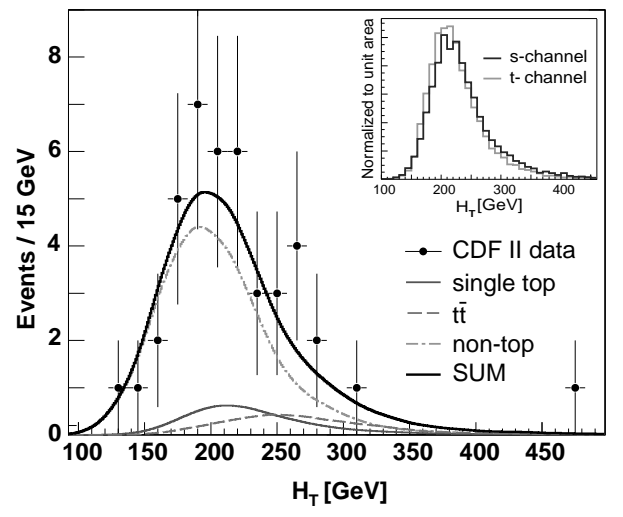

Figure 2: $H_{T}$ distribution for data (42 events) in the combined search compared with smoothed MC predictions for signal and background.

\begin{tabular}{|l|c|c|c|}
\hline & t-channel & s-channel & Combined \\
\hline expected limit & 11.2 & 12.1 & 13.6 \\
observer limit & 10.1 & 13.6 & 17.8 \\
MPV \pm HPD & $0.0_{-0.0}^{+4.7}$ & $4.6_{-3.8}^{+3.8}$ & $7.7_{-4.9}^{+5.1}$ \\
\hline
\end{tabular}

Table 2: Upper limits at 95\% confidence level and Most Probable Value and Highest Posterior Density intervals of the single top cross section in pb.

background events. One important variable to discriminate against $W+$ light-quark-jets will be the output of an advanced Neural Net $b$ tagger. The systematic uncertainties associated with modeling the signal will be extracted from comparisons to the NLO calculations. With these methods, we project a first evidence for Standard Model single top production with $\sigma=3$ for $1.5 \mathrm{fb}^{-1}$, excluding systematic uncertainties.

\section{References}

[1] T. Stelzer, Z. Sullivan, S.S. Willenbrock, Phys. Rev. D 56, 5919 (1997); M.C. Smith and S.S. Willenbrock, Phys. Rev. D 54, 6696 (1996); S. Mrenna and C.-P. Yuan, Phys. Lett. B 416, 200 (1998).

[2] B.W. Harris, E. Laenen, L. Phaf, Z. Sullivan, S. Weinzierl, Phys. Rev. D 66, 054024 (2002); Z. Sullivan, hep-ph/0408049.

[3] Levan Babukhadia et al. (CDF and DØ Higgs Working Group), FERMILAB-PUB-03-320-E. Phys. Lett. B 517, 282 (2001); Phys. Rev. D 63, 031101 (2000).

[4] The polar angle $\theta$ is measured w.r.t. the proton beam direction. The pseudorapidity is defined as $\eta \equiv-\ln \tan (\theta / 2) . E_{T}=E \sin (\theta)$.

[5] CDF Collaboration, FERMILAB-PUB-96/390-E (1996).

[6] D. Acosta et al. [CDF Collaboration], Phys. Rev. D 71, 012005 (2005) [arXiv:hep-ex/0410058]. 